\title{
A fish activity-monitoring device using a low-power infrared photobeam
}

\author{
THOMAS G. UTER \\ Department of Neurosciences, School of Medicine \\ and Neurobiology Unit, Scrips Institution of Oceanography \\ University of California at San Diego, MS A-001, La Jolla, California 92093
}

\begin{abstract}
An activity-monitor system for fish or other aquatic animals has been developed that uses a single low-level infrared (IRED) photobeam. The IRED source emits with a peak wavelength of $960 \mathrm{~nm}$ and requires only $70-\mathrm{mW}$ average power input for an operating range of greater than $50 \mathrm{~cm}$ in sea water. This represents an order of magnitude reduction in power and more than a doubling of range over other reported designs. The cost for a single-beam channel is approximately $\$ 30$.
\end{abstract}

Devices to monitor the activity of aquatic animals, particularly fish, both marine and fresh water, have taken many forms, including (1) tethering the animal to an electromechanical position sensor, (2) electromechanical systems that require the animal to physically displace a door, flap, or other obstacle in order to pass from one compartment to another (Davis, 1963; Spoore, 1941), (3) ultrasonic or doppler-shift movement detection (Byrne, 1971; Cummings, 1963; Meffert, 1968), (4) magnetic field or inductive movement detection (Schuyf \& de Groot, 1971), (5) time-lapse cinematography (Bainbridge, 1958; Webb, 1970), (6) closed-circuit TV/computer analysis (Uter, 1977), (7) detection of the changes in thermal absorption characteristics at a solid-liquid interface as affected by movement (Northmore \& Muntz, 1974), (8) detection of distortion or interruption of a photobeam (Cripe, Cripe, \& Livingston, 1975; Hafeez \& Baber, 1976; Kleerekoper, Timms, Westlake, Davy, Malar, \& Anderson, 1970; Parker, 1975), and (9) using photocells to monitor shadows cast against a screen by the moving animal (Hudson \& Busseil, 1972).

To study the diurnal characteristics of the marine fish Oxyijulius californica (Senorita fish) under the effects of various experimental alterations, the following requirements for an activity monitor were established: (1) no training of the animal; (2) no mechanical or package attachments to the animal; (3) not affected by changes in ambient light level; (4) two or more monitors must operate in the same aquarium, which has been divided into multiple compartments with each compartment containing one fish; (5) must not alter the "normal" behavior of the fish; (6) inexpensive to construct; (7) operate over a distance of at least $40 \mathrm{~cm}$ in sea water; $(8)$ be compact so that 10 experimental

This work was supported by grants to T. H. Bullock from the National Science Foundation and the National Institute of Health. aquaria plus detectors can fit into an existing relatively small space; and (9) be capable of driving an event recorder directly.

In matching up the list of requirements with the advantages and disadvantages of the existing techniques, it became apparent that the method of photobeam interruption had the most likelihood of satisfying all needs. Furthermore, based on the work of Chaston (1968) and Northmore and Muntz (1974), there was good evidence to support a choice of an infrared detector system.

\section{METHOD}

Following the work of Cripe et al. (1975), we decided to employ an IRED photodiode source and phototransistor detector operating in the $960-\mathrm{nm}$ wavelength region. However, the Cripe et al. design proved unsatisfactory for our use, owing to its limited $20-\mathrm{cm}$ range.

To produce the needed $40-\mathrm{cm}$ range, a synchronous sourcedetector design was chosen (Sahm, 1976). This technique has several inherent advantages over the more common continuous wave (CW) approaches. One such advantage is that the IRED source can be pulsed at 5 to 10 times the $C W$ power levels, while maintaining the average power at much lower levels. In the Cripe et al. design, each IRED source must dissipate about $600-\mathrm{mW}$ average power. In our design, the source dissipates only $60-\mathrm{mW}$ average power. Second, operating at higher instantaneous photoemission levels extends the distance the photobeam travels before being attenuated to undetectable levels. A third advantage is that the system inherently averages over time and thus, shortterm perturbances in the IRED source output are ignored. Finally, being synchronous, the detector is relatively insensitive to ambient light levels, particularly if these levels are produced by fluorescent lamps that are continuously turning on and off at $120 \mathrm{~Hz}$, since the synchronous oscillator can easily be set at a frequency that will cause the $120 \mathrm{-Hz}$ light source to average to zero in its effect on the system. On the other hand, incandescent or filament light sources produce a large amount of "dc" IRED radiation. Since the source is nearly constant in intensity, the monitor should not "see" this dc light source, as the monitor is an ac coupled amplifier. In practice, however, it is possible for a dc light source to drive the IRED detector phototransistor out of its correct bias conditions, thereby limiting its amplification properties. Although the peak response of the selected 

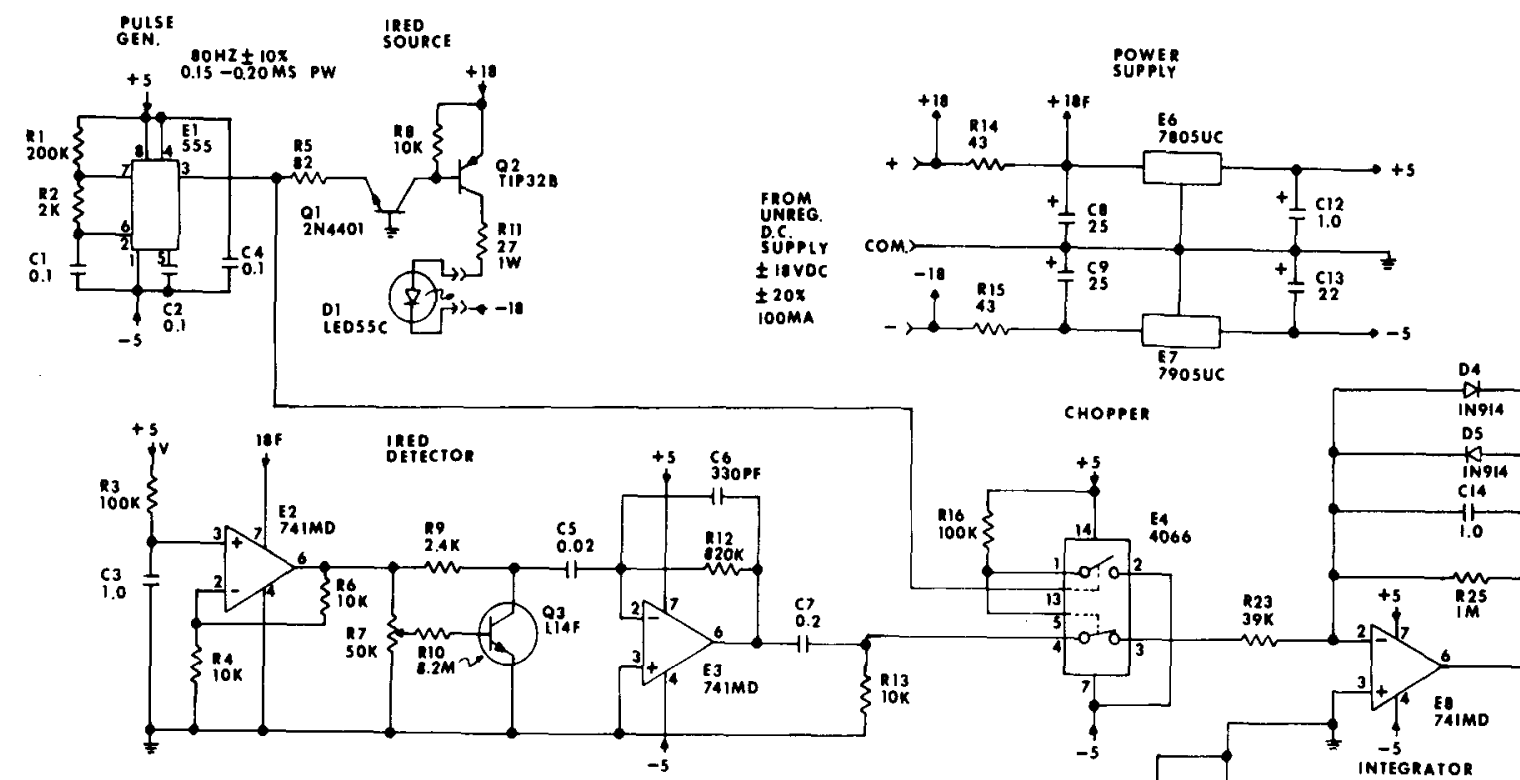
output is low, and the LED indicator D3 will be on. Q4 will be off and no current will flow through D2. When the photobeam is interrupted, the output will be high, and Q4 will be on, thus allowing current to flow in D2, which completes the event-recorder circuit.

\section{CONSTRUCTION}

To facilitate construction of 12 independent monitors, a printed circuit board was designed. Although crude (the art work was done using standard terminal pads interconnected by hand-drawn lines), it was relatively fast to develop and straightforward to manufacture. Prior to laying out the art work, several hand-wired prototypes were constructed.

If the user desires to hand wire an instrument, he should be aware that the IRED detector, Q3 in combination with E3, is a very high-gain current-tovoltage amplifier. Therefore, precautions should be taken to keep it well isolated from the IRED source circuitry. The design of the power supply and the decoupling capacitor $\mathrm{C} 4$ associated with E1 electrically isolate the IRED detector. Keeping all leads associated with the IRED source separated by a minimum of $1 \mathrm{~cm}$ from those of the IRED detector decreases the possibility of cross talk between the two circuits.

Since our system is required to "see" from one side of an aquarium to the other, D1 is mounted at the end of a two-conductor shielded cable of suitable length (the shield is grounded). Because of the very low power dissipated within the IRED source, no heat sinking is required. The remainder of the instrument is mounted in an aluminum box that serves as an E-field shield and light shroud.

A plastic bracket holds the IRED source in place on the aquarium side opposite the receiver box. The IRED beam is admitted to the receiver box through a small hole in the box cover covered by a Wratten 92 red filter. The filter minimizes the effects of ambient background-light levels as explained earlier.

Both R20 and D3 are mounted on the top of the receiver box to facilitate set-up. Terminals for connecting to a remotely located event recorder are also provided. A long length of two-conductor shielded cable is hardwired to the receiver box to bring in power from the unregulated dc supply. To minimize corrosion, the aluminum receiver box is sealed in a plastic bag. Adjustment of R20 is made with the bag in place.

\section{ADJUSTMENTS}

At initial start-up, R7 is adjusted so that the collector voltage of Q3 is between 4.5 and $5.5 \mathrm{~V}$ dc. This adjustment is carried out with Q3 exposed to the ambient-light operating conditions, but not to the IRED source. In our application, the ambient light is a 24-h light-dark cycle. R7 was adjusted under dark ambient, then checked for differences in the operation of the system in light ambient. No differences were found.

R20 sets the event threshold; it is set empirically in combination with the physical alignment of the IRED source and detector. For initial checkout, holding the beam axis of the IRED source at right angles to the axis of the detector transistor with a separation distance of $15 \mathrm{~cm}$ simulates normal operating conditions without requiring the use of a sea water tank. R20 is adjusted one rotation (in the direction of keeping D3 on) past the point where D3 just lights with no object interrupting the photobeam. During installation, R20 is further trimmed to provide suitable system operation using an opaque object to interrupt the photobeam. Finally, a fish is introduced into the tank.

\section{MODIFICATIONS}

Only a single photobeam has been implemented in this design. Addition of more beams can be accomplished without increasing power supply requirements, since additional IRED sources may be placed in series with the existing IRED source, and R11 reduced. One must, of course, add additional detectors. The outputs of each detector may be "wired OR'd" at the collector of Q4, or summed and integrated by E8. In the latter case, the interruption of any one beam would be sufficient to lower the integrated output below the required threshold.

\section{PERFORMANCE}

Tests show that objects as small as a standard pencil (6-mm diam) interrupt the photobeam at either side of a $50-\mathrm{cm}$-wide aquarium filled with sea water. Because the particular fish being studied are relatively large and slow moving, circuit time constants require that the photobeam be interrupted for $2 \mathrm{sec}$ before the leading edge of an event is recorded. The beam must be reestablished for $1 \mathrm{sec}$ for the trailing edge of the event to be recorded.

\section{REFERENCES}

Bainbridge, $R$. The speed of swimming fish as related to size and to the frequency and amplitude of the tail beat. Journal of Experimental Biology, 1958, 35, 109-133.

BYRNE, J. E. A further contribution to using ultrasonic sensors for fish activity studies. Transactions of the American Fisheries Society, 1971, 4, 792-794.

Chaston, J. Influence of light on activity of brown trout (Salmo trutta). Journal of the Canadian Fisheries Research Board, 1968, 25, 1285-1289.

Cripe, C. R., Cripe, J. H., \& Livingston, R. J. Apparatus for the quantitative determination of locomotor activity patterns of aquatic organisms using infrared light-emitting diodes. Journal of the Canadian Fisheries Research Board, $1975,32,1884-1886$. 
Cummings, W. C. Using the doppler effect to detect movements of captive fish in behavior studies. Transactions of the American Fisheries Society, 1963, 92, 178-180.

DAvis, R. E. Daily predawn peak of locomotion in blue-gill and largemouth bass. Animal Behaviour, 1963, 12 , $272-283$.

HAFEez, M. A., \& BaBER, B. J. A method for monitoring spontaneous locomotor activity and resting behavior in fishes. Physiology and Behavior, 1976, 16, 217-221.

Hudson, R. C. L., \& Bussell, D. M. A simple photoelectric movement monitor. Electroencephlography and Clinical Neurophysiology, 1972, 32, 445-447.

Kleere koper, H., Timms, A. M., Westlake, G. F., Davy, F. B., Malar, T., \& Anderson, V. M. An analysis of locomotor behaviour of goldfish (Carassius auratus). Animal Behaviour, 1970, 18, 317-330.

MEFFERT, P. Ultrasonic recorder for locomotor activities studies. Transactions of the American Fisheries Society, $1968,97,12-17$.

Northmore, D. P. M., \& Muntz, W. R. A. Effects of stimulus size on spectral sensitivity in fish (Scardinius erythrophthalmus), measured with a classical conditioning paradigm. Vision Research, 1974, 14, 503-514.

Parker, N. C. Activity patterns, feeding and behavior of the pirateperch Aphredoderus sayanus. Copeia, 1975, 3, 572-574.

SAHM, W. H. General Electric optoelectronics manual. Syracuse, New York: General Electric, 1976.

SChUYF, A., \& DE GROOT, S. J. An inductive locomotion detector for use in diurnal activity experiments in fish. Journal de Conseil, 1971, 34, 126-131.

SPOORE, W. A. A method of measuring the activity of fish. Ecology, 1941, 22, 329.331.

UTER, T. G. A real-time video system for tracking onedimensional movements of two objects. IEEE Transactions on Biomedical Engineering, 1977, 24, 75-78.

WEBB, P. W. Some aspects of the energies of swimming of fish with special reference to the cruising performance of rainbow trout Salmo gairdneri Richardson. Unpublished Ph.D. thesis, Bristol University, Bristol, England, 1970.

(Received for publication March 17, 1978; revision accepted April 17, 1978.) 